\title{
Surgery and Hepatocellular Carcinoma
}

\author{
Nobuhisa Akamatsua Umberto Cillob Alessandro Cucchettic \\ Matteo Donadon ${ }^{d}$ Antonio Daniele Pinna ${ }^{c}$ Guido Torzillid \\ Norihiro Kokudo ${ }^{a}$ \\ aHepato-Biliary-Pancreatic Surgery Division, Artificial Organ and Organ Transplantation Division, \\ Department of Surgery, Graduate School of Medicine, University of Tokyo, Tokyo, Japan, bUnità di \\ Chirurgia Epatobiliare e Trapianto Epatico, Azienda Ospedaliera, Università di Padova, Padova, \\ 'Department of Medical and Surgical Sciences, DIMEC; S. Orsola, Malpighi Hospital; Alma Mater \\ Studiorum, University of Bologna, Bologna, dDepartment of Surgery, Division of Hepatobiliary and \\ General Surgery, Humanitas University and Humanitas Research Hospital, Milan, Italy
}

\author{
Key Words \\ Hepatocellular carcinoma · Liver resection · Liver transplantation
}

\begin{abstract}
The optimal surgical strategy for hepatocellular carcinoma (HCC) is under active debate. Biomarkers of the liver functional reserve as well as volumetric analysis of the future liver remnant are essential for safe liver resection of HCC. The present algorithms applied to surgical strategies for HCC are not ideal because many patients who could potentially undergo safe resection are deemed liver transplant candidates in Western countries, whereas the opposite is the case in Eastern countries. In addition, there is too much focus on expanded criteria for patients with HCC to undergo liver transplantation. The transplantation benefit for patients with HCC should be considered based not only on the individual's benefit, but also on the effect of other patients waiting for LT for other indications. $\quad$ Copyright $\odot 2016 \mathrm{~S}$. Karger AG, Basel
\end{abstract}

\section{Introduction}

The optimal surgical strategy for hepatocellular carcinoma (HCC) is an ongoing debate. When considering liver resection for HCC, the extent of radical resection required to resolve the tumor burden, as well as the functional reserve of the diseased liver, and the volume of 
the future liver remnant must be taken into account. In addition, liver transplantation (LT), which can potentially cure both the diseased liver and HCC, is now an established surgical treatment for patients with HCC. Numerous methods for estimating the liver functional reserve and algorithms for surgical strategy in the treatment of HCC are available. This review discusses the current opinions regarding resection and LT for HCC.

\section{Liver Resection}

\section{Selection Criteria for Safe Hepatectomy.}

The required remnant liver volume depends on the liver function in those with chronic liver dysfunction, as proposed by Torzilli et al [1]; the worse liver function is, the smaller the liver volume that can be resected safely. For example, 30\% liver remnant may be sufficient in patients with normal liver function, but a higher percentage of liver remnant is recommended when baseline liver function is abnormal. The most reliable method for estimating the liver functional reserve has not yet been clarified. This uncertainty is in respect of more HCC sufferers who are mostly cirrhotic, because operative mortality is still significant in some series [2]. There are several methods for estimating the liver functional reserve which include the indocyanine-green retention rate at 15 minutes, $99 \mathrm{mTc}$-galactosyl human serum albumin scintigraphy, and ${ }^{13} \mathrm{C}$-Methacetin Breath Test (LiMAx ${ }^{\circledR}$ ). Other methods include scoring systems, such as the Child-Pugh-Turcotte (CPT), the Model for End-stage Liver Disease (MELD), and the aspartate transaminase-to-platelet ratio index (APRI). The CPT scoring system is popular, but it does not allow for the stratification of class A patients on who surgery is focused. Application of the MELD score to decide whether to undergo liver resection has produced disparate results $[2,3]$. Similar to the CPT score, the MELD score works well for patients with marginal liver function, but it fails to differentiate the surgical risk in those patients in which HCC is more suitable for resection. While the APRI score is promising, there are few reports of discussing APRI score [4]. Similar to the CPT and MELD scores, the APRI score seems adequate for patients with advanced liver disease, i.e. patients with high aspartate transaminase and/or thrombocytopenia, who are preferentially nonsurgical. The role of serum bilirubin and serum cholinesterase in predicting postoperative outcome was recently established based on approximately 400 consecutive hepatectomies for HCC. While CPT, MELD, and APRI scores did not significantly affect the short-term outcome, the serum bilirubin ( $>1 \mathrm{mg} / \mathrm{dl}$ or $17.1 \mu \mathrm{mol} / \mathrm{L})$ and cholinesterase $(<5900 \mathrm{UI} /)$ values were significantly associated with postoperative morbidity. Consequently, a scoring system, named the serum bilirubin/serum cholinesterase (BILCHE) score, was devised [5]. Promising results are expected with the combined application of the BILCHE score and FibroScan ${ }^{\circledR}$ [6]. In conclusion, the BILCHE score may be helpful for surgeons in selecting patients awaiting surgery for HCC to identify those at greater risk of postoperative complications.

\section{Liver Surgery in the Real World}

Liver surgery has become an established therapy for patients with HCC and cirrhosis. However, surgeons and oncologists disagree in regard to the tumor burden suitable for surgical removal for adequate survival. Indeed, in the current European Association for the Study of the Liver-European Organisation for Research and Treatment of Cancer guidelines [7], surgery is restricted to those patients in the very early or early stages of disease (BarcelonaClinic Liver Cancer score [BCLC] 0-A). Several studies have demonstrated a potential role of surgery for patients with large multinodular, and macrovascular invasive HCC (BCLC stage $\mathrm{B}$ and $\mathrm{C}$ ). A recent multicenter study reported that the 5-year overall survival of patients in 


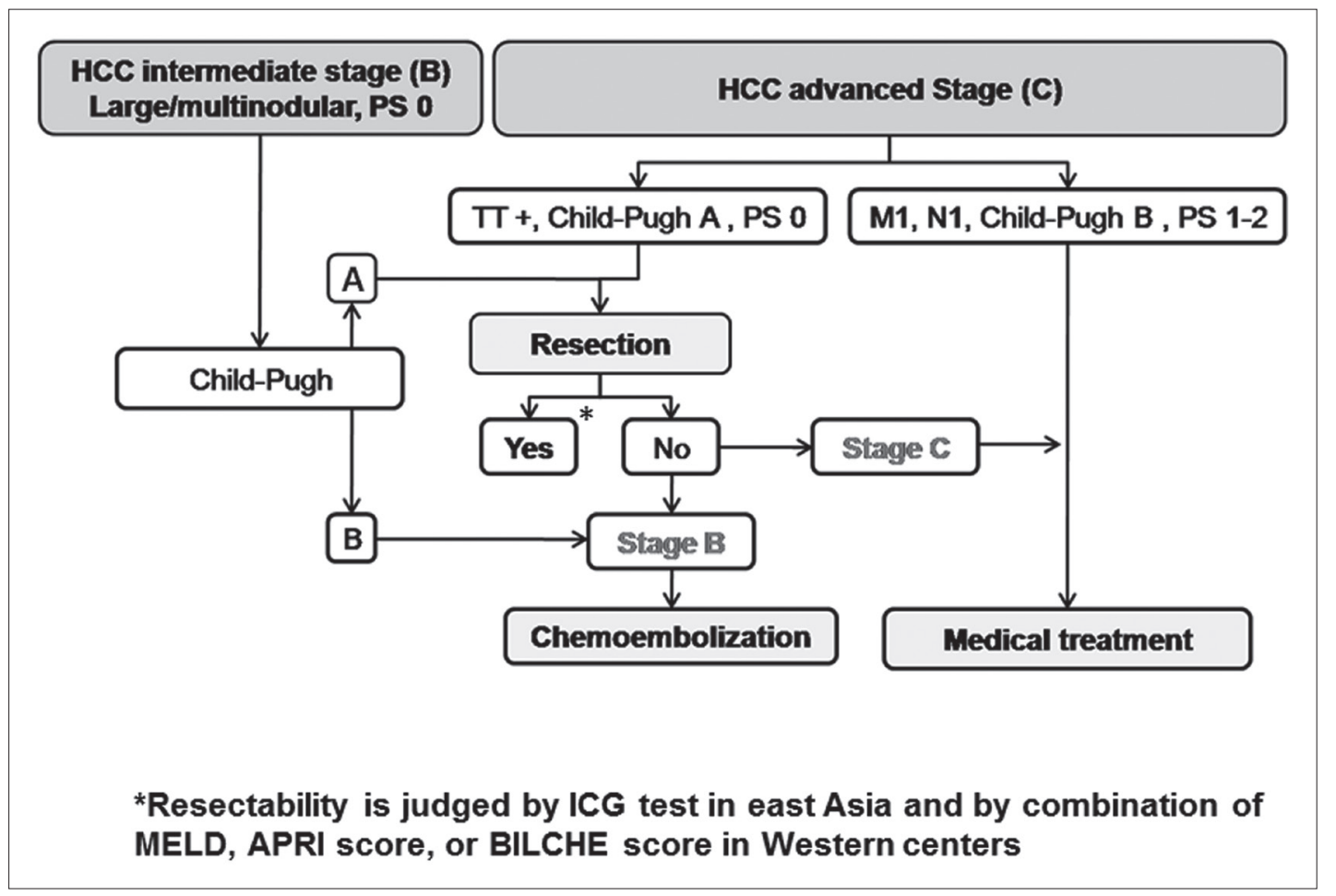

Fig. 1. Modified Barcelona-Clinic Liver Cancer algorithm. $\mathrm{TT}=$ tumor thrombus; $\mathrm{PS}=$ performance status; $\mathrm{M}=$ metastases; $\mathrm{N}=$ nodes (lymph nodes); $\mathrm{ICG}=$ indocyanine green.

BCLC stages B and C had 57\% and 38\% overall survival, respectively [8]. However, the study was criticized as biased due to the misinterpretation of stage $B$, which should have included only those patients with multinodular HCC, and not those with single HCC nodules larger than $5.0 \mathrm{~cm}$. Even in the most recent literature, the classification system lacks clarity $[7,9,10]$, as in one study stage B included those patients with single HCC nodules larger than $5 \mathrm{~cm}$ [7], and another study did not [9]. Conversely, the rationale based on which large HCC, if not intermediate, can be defined as early, should be clarified. Recent articles on this issue supported the inclusion of large HCC within stage B $[10,11]$. In any case, whether or not large HCC should be included in stage B, the aforementioned multicenter study [8] reported that $50 \%$ of patients with intermediate or advanced stage HCC are treated routinely with surgery in tertiary referral centers worldwide. These reports support the notion that surgery is not just an anecdotal solution for these patients, and the remarkable survival as a result does not justify the former exclusion of surgery in preference for other available therapies. Thus, surgery should at least be considered in a multidisciplinary setting as a potentially curative therapy for patients with BCLC stages B and C. As a result, the therapeutic flow-chart for these patients should be modified as shown in fig. 1.

\section{LT}

\section{The Western Perspective}

LT is the only treatment that offers the real chance of a cure for both HCC and underlying liver cirrhosis [12]. However, the shortage of liver grafts and the possibility of tumor 
recurrence are strong limiting factors. Grafts in Western countries are primarily obtained from cadaveric donors and organ allocation is the responsibility of each country's healthcare system, which applies several different rules based on liver functional reserve and tumor burden [13]. Two main aspects must be considered in these candidates namely: 1) appropriate recipient selection criteria, aimed at reducing posttransplant tumor recurrence to a minimum, and 2) fairness among candidates with and without HCC. Several studies support the proposal that the commonly accepted Milan criteria can be safety expanded with an acceptable postoperative outcome. However, this aspect must be weighed against the biology of the tumor, which has a pivotal role in determining posttransplant tumor relapse. Recently, including biomarkers such as alpha-fetoproteinand des-gamma carboxyprothrombin into the criteria have been reported to improve the performance of recipient selection [14]. Patients with down-staged tumors have excellent survival after transplantation and a complete response to neoadjuvant treatments is a reflection of less aggressive tumor behavior. Such selection criteria, even with the aid of tumor biopsy, can allow for transplantation in more patients without negatively affecting survival [14]. The second important aspect is in regard to fairness in accessibility to LT for patients with and without HCC. Patients with HCC represent a considerable proportion of candidates in Western countries, especially in Europe, whose urgency is not appreciated by the MELD score because of frequently preserved liver function. For this reason, additional MELD scores have been added for HCC candidates to increase their priority ranking [13]. The main drawback when giving priority on the basis of tumor stage is that an allocation system unbalanced towards such urgency can compromise utility. Indeed, the higher the priority score for patients with HCC, the poorer the posttransplant outcome [15]. To overcome this dilemma, the solution probably lies in the concept of a transplant benefit aimed at restoring equity to the allocation system by equalizing the transplant benefit, and not pre- or posttransplant survival, between HCC and non-HCC candidates $[13,16]$. Future studies should aim to build a strong transplant benefit model that is widely validated and adopted in the real clinical setting.

\section{The Eastern Perspective}

LT has become an established treatment for cirrhotic patients with HCC. Due to the severe organ shortage, living-donor liver transplantation (LDLT) has become mainstream in Eastern countries, with results that are comparable to those obtained by cadaveric-donor liver transplantation (CDLT) in Western countries [17]. Unlike CDLT, LDLT is not limited by the restrictions imposed by the nationwide allocation system, and the indication for LDLT in patients with HCC often depends on institutional or case-by-case considerations, balancing the burden on the donor, the operative risk, and the overall survival benefit for the recipient [18]. In terms of liver function, the indication for LT in the treatment algorithm for HCC differs significantly among Eastern countries $[18,19]$, from permitting such surgery in CPT stage A patients in Hong Kong [20], to restricting it to only CPT stage C patients in Japan [21]. In terms of tumor burden, the Milan criteria are recommended in all algorithms, but actual clinical practice seems to differ among countries. In Japan, while national insurance covers only those within the Milan criteria, many high-volume centers perform LDLT for those beyond the Milan criteria with their own unique criteria. Similarly, in Korea, the indication of LDLT for HCC is center-oriented, but the insurance system covers all LT for HCC. Taiwan and Hong Kong use the University of California, San Francisco (UCSF) criteria. Accumulating data from Eastern perspectives indicate that extending the Milan criteria may be valid, especially in the LDLT setting where the allocation impact on those with other diseases is absent [18]. At the same time, given that Eastern countries have excellent locoregional treatment strategies for HCC, LT should be avoided for those who can expect comparable long-term survival 
by the former treatments. In conclusion, LDLT will continue to be a mainstay treatment for HCC in cirrhotic patients with promising outcomes expected in Eastern countries.

\section{The Transplant Benefit as an Endpoint}

The Milan criteria and surrogate markers, in the selection of patients with HCC for LT represent a paradigm based purely on achieving high posttransplant long-term survival (posttransplant utility). Such a paradigm is too simplistic and is now the object of a number of criticisms for the following reasons:

PROBLEM 1: The "posttransplant utility" endpoint does not capture pretransplant patient loss for dropout whilst on the waiting list. Indeed, the transplant process is not described with an intention-to-treat perspective, therefore preventing a comparison of transplant results with data from alternative treatments such as resection or ablation.

PROBLEM 2: The Milan criteria and surrogate markers may allow inclusion on the transplant list of a number of patients at low need for transplantation. These criteria do not take into consideration the therapeutic alternatives available to the patients (e.g. resection, ablation, and others).

PROBLEM 3: Due to their predictive inaccuracy, macromorphologic parameters may exclude a relevant number of patients with a potential for acceptable posttransplant results.

PROBLEM 4: The same waiting list includes patients listed according to an endpoint of urgency (MELD - patients with cirrhosis) and patients listed according to an endpoint of utility (Milan criteria - patients with HCC). Therefore, an endpoint disquiet occurs among different patient groups.

The transplant benefit as an endpoint for patient selection and patient prioritization may solve these problems, representing a more efficient method in LT management. The transplant benefit can be calculated as the area under the curve between intention-to-treat expected for survival with LT (survival expected from the moment of listing) versus the expected intentionto-treat for survival with the best alternative therapy available (e.g. resection, ablation, and others). It can be expressed as gain in life expectancy between the two options considered (gain in quality adjusted life years).

The transplant benefit as an endpoint has relevant advantages as it is able to capture: 1) the real need for LT according to the available alternative therapies, 2) the overall efficiency of the LT process, including waiting list management and dropout rate (intention-to-treat analysis), 3) the quality of the selection criteria, donor-recipient matching, perioperative, and long-term management (the greater the short- and long-term survival rates, the greater the transplant benefit), and 4) the cost effectiveness of LT (relation between LT costs and qualityadjusted life years gained).

Some evidence for the transplantation benefits of LT was recently presented. In a Markov model, we demonstrated that the transplant benefit is higher in patients with HCC with a partial response to first-line non-transplant therapies [22]. Barry et al. calculated that the gain in life expectancy of LT over resection is only 2.8 months after 5 years [23]. We confirmed these findings, calculating the expected gain in life expectancy of LT versus non-transplant therapies for patients stratified by BCLC stage. We found that in patients with BCLC stage A the 5-year transplant benefit was only 13.5 months, whereas it was 17.4 months in patients with BCLC stage B or C, and 28.5 months in patients with BCLC stage D [24].

Together these lines of evidence suggest that, while more accurate predictors of the transplant benefit are needed, some HCC patient subpopulations with a clearly greater transplant benefit, can be identified. These patients deserve priority in the transplantation selection process [25]. Namely, patients with decompensated cirrhosis (BCLC stage D), successful down-staging, or with a recurrence or a partial response to a non-transplant therapy all have an intrinsically high transplant benefit due to their poor long-term expected survival without 
LT. Of course, these patients with a potentially high transplant benefit must be "filtered" according to the accepted utility criteria (Milan, UCSF, total tumor volume, and others) to ensure acceptable post-LT long-term survival. The Italian liver transplant community recently published a new allocation model diffusely adopting the transplant benefit endpoint [26]. However, further studies are needed.

\section{Conclusion}

The optimal surgical treatment for HCC based on the tumor burden, liver functional reserve, and availability of liver grafts for LT remains to be established.

\section{Disclosure Statement}

All authors declared no conflict of interest.

\section{References}

1 Torzilli G, Makuuchi M, Inoue K, Takayama T, Sakamoto Y, Sugawara Y, Kubota K, Zucchi A: No-mortality liver resection for hepatocellular carcinoma in cirrhotic and noncirrhotic patients: is there a way? A prospective analysis of our approach. Arch Surg 1999;134:984-992.

2 Teh SH, Christein J, Donohue J, Que F, Kendrick M, Farnell M, Cha S, Kamath P, Kim R, Nagorney DM: Hepatic resection of hepatocellular carcinoma in patients with cirrhosis: Model of End-Stage Liver Disease (MELD) score predicts perioperative mortality. J Gastrointest Surg 2005;9:1207-1215, discussion 1215.

3 Cucchetti A, Ercolani G, Vivarelli M, Cescon M, Ravaioli M, La Barba G, Zanello M, Grazi GL, Pinna AD: Impact of model for end-stage liver disease (MELD) score on prognosis after hepatectomy for hepatocellular carcinoma on cirrhosis. Liver Transpl 2006;12:966-971.

4 Ichikawa T, Uenishi T, Takemura S, Oba K, Ogawa M, Kodai S, Shinkawa H, Tanaka H, Yamamoto T, Tanaka S, Yamamoto S, Hai S, Shuto T, Hirohashi K, Kubo S: A simple, noninvasively determined index predicting hepatic failure following liver resection for hepatocellular carcinoma. J Hepatobiliary Pancreat Surg 2009;16:42-48.

5 Donadon M, Costa G, Cimino M, Procopio F, Fabbro DD, Palmisano A, Torzilli G: Safe hepatectomy selection criteria for hepatocellular carcinoma patients: a validation of 336 consecutive hepatectomies. The BILCHE score. World J Surg 2015;39:237-243.

6 Cescon M, Colecchia A, Cucchetti A, Peri E, Montrone L, Ercolani G, Festi D, Pinna AD: Value of transient elastography measured with FibroScan in predicting the outcome of hepatic resection for hepatocellular carcinoma. Ann Surg 2012;256:706-712; discussion 712-703.

7 European Association For The Study Of The Liver European Organisation For Research And Treatment Of Cancer: EASL-EORTC clinical practice guidelines: management of hepatocellular carcinoma. J Hepatol 2012;56:908-943.

8 Torzilli G, Belghiti J, Kokudo N, Takayama T, Capussotti L, Nuzzo G, Vauthey JN, Choti MA, De Santibanes E, Donadon M, Morenghi E, Makuuchi M: A snapshot of the effective indications and results of surgery for hepatocellular carcinoma in tertiary referral centers: is it adherent to the EASL/AASLD recommendations?: an observational study of the HCC East-West study group. Ann Surg 2013;257:929-937.

9 Forner A, Llovet JM, Bruix J: Hepatocellular carcinoma. Lancet 2012;379:1245-1255.

10 Torzilli G, Belghiti J, Kokudo N, Takayama T, Capussotti L, Nuzzo G, Vauthey JN, Choti MA, De Santibanes E, Donadon M, Makuuchi M: Reply to Letter: "Dissecting EASL/AASLD Recommendations With a More Careful Knife: A Comment on 'Surgical Misinterpretation' of the BCLC Staging System”: Real Misinterpretation or Lack of Clarity Within the BCLC? Ann Surg 2015;262:e18-e19.

11 Jung YK, Jung CH, Seo YS, Kim JH, Kim TH, Yoo YJ, Kang SH, Yim SY, Suh SJ, An H, Yim HJ, Yeon JE, Byun KS Um SH: BCLC stage B is a better designation for single large hepatocellular carcinoma than BCLC stage A. J Gastroenterol Hepatol 2016:31:467-474.

12 Cucchetti A, Vitale A, Cescon M, Gambato M, Maroni L, Ravaioli M, Ercolani G, Burra P, Cillo U, Pinna AD: Can liver transplantation provide the statistical cure? Liver Transpl 2014;20:210-217.

13 Toso C, Mazzaferro V, Bruix J, Freeman R, Mentha G, Majno P: Toward a better liver graft allocation that accounts for candidates with and without hepatocellular carcinoma. Am J Transplant 2014;14:2221-2227. 
14 Chan SC, Fan ST: Selection of patients of hepatocellular carcinoma beyond the Milan criteria for liver transplantation. Hepatobiliary Surg Nutr 2013;2:84-88.

15 Cucchetti A, Cescon M, Bertuzzo V, Bigonzi E, Ercolani G, Morelli MC, Ravaioli M, Pinna AD: Can the dropout risk of candidates with hepatocellular carcinoma predict survival after liver transplantation? Am J Transplant 2011;11:1696-1704.

16 Vitale A, Volk ML, De Feo TM, Burra P, Frigo AC, Ramirez Morales R, De Carlis L, Belli L, Colledan M, Fagiuoli S, Rossi G, Andorno E, Baccarani U, Regalia E, Vivarelli M, Donataccio M, Cillo U, Liver Transplantation North Italy Transplant program (NITp) working group: A method for establishing allocation equity among patients with and without hepatocellular carcinoma on a common liver transplant waiting list. J Hepatol 2014;60:290-297.

17 Akamatsu N, Sugawara Y, Kokudo N: Living-donor vs deceased-donor liver transplantation for patients with hepatocellular carcinoma. World J Hepatol 2014;6:626-631.

18 Akamatsu N, Sugawara Y, Kokudo N: Living donor liver transplantation for patients with hepatocellular carcinoma. Liver Cancer 2014;3:108-118.

19 Han KH, Kudo M, Ye SL, Choi JY, Poon RT, Seong J, Park JW, Ichida T, Chung JW, Chow P, Cheng AL: Asian consensus workshop report: expert consensus guideline for the management of intermediate and advanced hepatocellular carcinoma in Asia. Oncology 2011;81(Suppl 1):158-164.

20 Yau T, Tang VY, Yao TJ, Fan ST, Lo CM, Poon RT: Development of Hong Kong Liver Cancer staging system with treatment stratification for patients with hepatocellular carcinoma. Gastroenterology 2014;146:16911700

21 Kokudo N, Hasegawa K, Akahane M, Igaki H, Izumi N, Ichida T, Uemoto S, Kaneko S, Kawasaki S, Ku Y, Kudo M, Kubo S, Takayama T, Tateishi R, Fukuda T, Matsui O, Matsuyama Y, Murakami T, Arii S, Okazaki M, Makuuchi M: Evidence-based Clinical Practice Guidelines for Hepatocellular Carcinoma: The Japan Society of Hepatology 2013 update (3rd JSH-HCC Guidelines). Hepatol Res 2015;45 doi: 10.1111/hepr.12464.

22 Cillo U, Vitale A, Volk ML, Frigo AC, Grigoletto F, Brolese A, Zanus G, D’Amico F, Farinati F, Burra P, Russo F, Angeli P, D'Amico DF: The survival benefit of liver transplantation in hepatocellular carcinoma patients. Dig Liver Dis 2010;42:642-649.

23 Berry K, Ioannou GN: Are patients with Child's A cirrhosis and hepatocellular carcinoma appropriate candidates for liver transplantation? Am J Transplant 2012;12:706-717.

24 Vitale A, Morales RR, Zanus G, Farinati F, Burra P, Angeli P, Frigo AC, Del Poggio P, Rapaccini G, Di Nolfo MA, Benvegnù L, Zoli M, Borzio F, Giannini EG, Caturelli E, Chiaramonte M, Trevisani F, Cillo U, Italian Liver Cancer group: Barcelona Clinic Liver Cancer staging and transplant survival benefit for patients with hepatocellular carcinoma: a multicentre, cohort study. Lancet Oncol 2011;12:654-662.

25 Vitale A, Burra P, Frigo AC, Trevisani F, Farinati F, Spolverato G, Volk M, Giannini EG, Ciccarese F, Piscaglia F, Rapaccini GL, Di Marco M, Caturelli E, Zoli M, Borzio F, Cabibbo G, Felder M, Gasbarrini A, Sacco R, Foschi FG, Missale G, Morisco F, Svegliati Baroni G, Virdone R, Cillo U, Italian Liver Cancer (ITA.LI.CA) group: Survival benefit of liver resection for patients with hepatocellular carcinoma across different Barcelona Clinic Liver Cancer stages: a multicentre study. J Hepatol 2015;62:617-624.

26 Cillo U, Burra P, Mazzaferro V, Belli L, Pinna AD, Spada M, Nanni Costa A, Toniutto P, I-BELT (Italian Board of Experts in the Field of Liver Transplantation): A Multistep, Consensus-Based Approach to Organ Allocation in Liver Transplantation: Toward a "Blended Principle Model”. Am J Transplant 2015;15:2552-2561. 